\title{
Questioning the Quality of Thyroid Cancer Data: Thoughts from the Commission on Cancer and the National Cancer Database
}

\author{
Kathleen K. Thoburn, CTR, Donna M. Gress, RHIT, CTR, Katherine Mallin, PhD, Ashley Loomis, MPH, \\ David P. Winchester, MD, FACS, and Daniel P. McKellar, MD, FACS
}

American College of Surgeons, Commission on Cancer, National Cancer Database, Chicago, IL

Cancer registry data is useful for retrospective analysis of treatment patterns and outcome. It is therefore imperative that data submitted to cancer databases such as the National Cancer Database (NCDB) is as accurate and complete as possible. For most cancer types, patients undergo a single operation as part of the treatment of that cancer; however, thyroid cancer is unique because patients often undergo an initial thyroid lobectomy (TL) followed later by a completion total or subtotal thyroidectomy (TT) as indicated by the initial operative pathology results. As a result, cancer registrars abstracting data regarding patients with thyroid cancer would have to assess whether a second procedure was performed in order to provide accurate surgical data to the cancer database.

In a previous study using NCDB data, Kiernan et al. observed that patients with TL were receiving radioactive iodine treatment (RAI), which is not recommended for patients treated with TL. ${ }^{1}$ This led the authors to question the accuracy of the surgical codes being reported to national cancer surveillance systems for thyroid patients, and prompted their present study. Using data reported to the Tennessee Cancer Registry (TCR), Kiernan et al. conducted a review of thyroid surgery codes submitted to the TCR for the diagnosis years 2004 through 2011. Submitted surgery codes were deemed as correct or incorrect based on review of submitted textual documentation and by contacting the reporting facility to confirm the submitted

(C) Society of Surgical Oncology 2016

First Received: 16 August 2016; Published Online: 15 November 2016

K. K. Thoburn, CTR

e-mail: kthoburn@facs.org surgery code and review the first course of treatment for any subsequent thyroid procedures.

Of the $921 \mathrm{TL}$ cases reviewed, the authors identified $40 \%$ as being coded incorrectly. In $27 \%$ of cases, the TL was revised to TT and, in the remaining cases, the surgery codes were revised from one type of TL to another type of TL.The authors state that identified inaccuracies due to changing from one TL code to another highlights that the Facility Oncology Registry Data Standards (FORDS) manual has multiple codes for procedures that are vague or overlapping and that the definitions likely result in inaccurate coding.

The researchers interviewed some cancer registrars to determine the reasons for inaccurate coding and reported that in a single institution the majority of cases that had errant codes submitted were for patients who underwent a completion thyroidectomy after TL. The registrars hypothesized that the second specimen did not contain cancer and therefore they were not prompted by pathology to update the surgical code, and/or additional procedures were not identified at the time of record review for followup or treatment.

This study brings attention to, and prompts further discussion on, the quality of thyroid surgical coding included in national cancer surveillance systems. Several potential mechanisms to improve thyroid surgery coding have been suggested. As noted in the article, the NCDB is currently involved in a major revision of the FORDS manual. The findings in this article were shared with the FORDS Revision Project Thyroid expert panel for feedback on the current thyroid surgery codes. Please refer to Appendix B: Site-Specific Surgery Codes for Thyroid, page 418 of the current FORDS manual (FORDS: Revised for 2016), for a listing of surgical codes for thyroid and their respective 
descriptions. The expert panel determined that the statement by the authors that the current version of the FORDS manual includes codes that are 'vague or overlapping' and likely result in inaccurate coding is unsubstantiated; the surgical codes for TL verses TT are clear and mutually exclusive.

Due to the fact that thyroid cancer often requires a second procedure to remove any remaining thyroid tissue, the cancer registry community needs to be educated on the need to determine whether a subsequent completion thyroidectomy was performed after a TL and report this to the cancer database. Emphasis on utilizing all available resources for case finding should be made and not simply based on a pathology report that contains a diagnosis of cancer. For the cases included in this study, review of diagnosis codes and primary procedure codes found in medical record disease indices would have identified the
TT, whereas review of a pathology report with no mention of cancer would not. In addition, thyroid data-quality edits need to be written to identify and prompt review of thyroid cases treated with TL that receive specific radiation treatment modalities. Finally, the Accreditation Committee of the Commission on Cancer has implemented a work group to improve the quality of NCDB data, including completeness, accuracy, reporting of recurrence, and follow-up of cancer cases.

\section{REFERENCE}

1. Kiernan CM, Parikh AA, Parks LL, Solorzano CC. Use of radioiodine after thyroid lobectomy in patients with differentiated thyroid cancer: does it change outcomes? J Am Coll Surg. 2015;220(4):617-25. 